\title{
The political economy of income taxation under asymmetric information: the two-type case
}

\author{
John E. Roemer
}

Received: 30 September 2010 / Accepted: 7 February 2011 / Published online: 2 March 2011 (C) The Author(s) 2011. This article is published with open access at SpringerLink.com

\begin{abstract}
The apparent ubiquity of progressive taxation in advanced democracies has animated research by political economists in the past decade, but little progress has been made in modeling political equilibria over tax policy when labor supply is elastic with respect to taxation. Here, we postulate an economy with two worker types (wage capacities), in which the unskilled are more numerous than the skilled. Preferences are quasi-linear in income and leisure. One political party represents each worker type. A tax policy is any feasible incentive-compatible menu of pre- and posttax incomes from which each worker must choose. This policy space is three-dimensional. Workers vote stochastically. The equilibrium concept for political competition is party-unanimity Nash equilibrium (PUNE) — thus, parties are both vote-seeking and representative. The set of political equilibria is characterized. We prove that, if the class of unskilled workers is not too large (but greater than one-half), then there always exist equilibria in which a regressive tax policy wins. If, however, that class is sufficiently numerous, or inequality is sufficiently great, then the victory of a progressive policy is guaranteed in all equilibria.
\end{abstract}

\footnotetext{
Although mass voting has not been a topic of Salvador Barberà's research, I offer this paper for this special issue in his honor, because it represents one of the unfortunately few attempts to bring the issue of voter incentive compatibility into the study of the politics of income re-distribution through taxation-and incentive compatibility has been the cornerstone of Salvador's research program. I emphasize 'politics,' because the optimal taxation literature, since Mirrlees (1971), has addressed incentive compatibility-but as a problem for the social planner. The present paper is an attempt to study Mirrleesian considerations in the context of political competition. I am grateful to an anonymous referee for acute comments on the first draft.
}

\footnotetext{
J. E. Roemer $(\varangle)$

Yale University, New Haven, USA

e-mail: John.roemer@yale.edu
} 
Keywords Progressive taxation · PUNE - Asymmetric information · Mirrlees tax problem

JEL Classification $\quad \mathrm{D} 72 \cdot \mathrm{H} 21$

\section{Introduction}

There have been, in the past decade, a significant number of contributions to the political economy of income taxation [Marhuenda and Ortuño-Ortin (1998), Gouveia and Oliver (1996), Roemer (1999), Carbonell-Nicolau and Klor (2003), De Donder and Hindriks (2003), Roemer (2006), Carbonell-Nicolau and Ok (2006), Casamatta et al. (2010), Roemer (2011), and Gomberg et al. (2010); in many of these papers, the focus has been on explaining the ubiquity of progressive income taxation. It is most natural to examine this problem in an environment where the policy space, from which parties choose an income-tax policy, is multi-dimensional. Because of this, equilibria in the classical Downsian model of political competition generally fail to exist.

My goal is to study the political competition over income-tax policies in a model which allows us to achieve four desiderata; that

(1) there be partisan parties, which represent different coalitions of constituents;

(2) worker-voters be characterized by different wage-earning capacities, where the parties know the distribution of such capacities, but the capacity of individuals is private information;

(3) worker-voters derive utility from income and leisure, and

(4) policies be unrestricted, except that they must be incentive compatible.

I have italicized the key requirements. None of the papers in the cited literature present models that achieve all these desiderata. Perhaps the desirability of (2)-(4) is self-evident. Nevertheless, most of the cited papers avoid the problem of incentive compatibility, by assuming that income is inelastically produced. I claim (1) is important as well, although it is not often implemented in the formal literature. An assumption frequently made by political economists, that parties are purely opportunist institutions that aim solely to maximize vote share, or their probability of victory, is unrealistic: in mature democracies, parties do not survive if they do not attempt to represent constituent interests. Indeed, the formal literature in this regard has been, in my view, insufficiently sensitive to non-formal political science, which is overwhelmingly concerned with studying parties that are, to some extent at least, institutions that represent coalitions of citizens.

In his classic paper, Mirrlees (1971) carried out a normative analysis of tax policy under assumptions (2)-(4). The present study is the beginning of an attempt to implement the Mirrlees program in a positive framework - that is, when political competition between parties representing citizen coalitions determines tax policy, rather than a benevolent government. The only other attempt along similar lines, of which I am aware, is the recent work of Boyer and Bierbrauer (2010).

The political equilibrium concept I use is party-unanimity Nash equilibrium (PUNE). This concept was introduced in Roemer (1999, 2001); it models parties as run by politicians who care both about winning and representation of constituents. 
In my earlier study of the politics of taxation, however, workers derived utility only from income, not leisure, and so the issue of incentive compatibility did not arise.

Working with a continuum of types, and demanding the desiderata 1 through 4 above, would pose an intractable problem. It would require solving two Mirrlees optimal tax problems, which are interdependent, as the reader will see below. ${ }^{1}$ I therefore opt to simplify the environment drastically, in order to be able to implement the declared desiderata.

After presenting some preliminaries in the next section, Sect. 3 defines political equilibrium, Sect. 4 characterizes the set of Pareto efficient policies and second-best efficient policies, Sect. 5 characterizes the set of political equilibria, and Sect. 6 discusses the extent to which victorious policies are redistributive or progressive.

\section{Preliminaries}

We assume that there are only two types of worker, characterized by different wage capacities, 1 and $\theta>1$, present in population fractions $p$ and $1-p$, where the unskilled type comprises a majority $(p>0.5){ }^{2}$ Let $\rho=\frac{p}{1-p}>1$. We assume as well that preferences are quasi-linear, represented by the utility function $u(x, L)=x-g(L), \quad x$ is income and $L$ is labor, where $g$ is a convex, increasing, continuously differentiable function, normalized so that $g(0)=0$. The labor (leisure) endowment of each worker is one. These two simplifying assumptions will render the problem analytically tractable.

The set of symmetric feasible income-labor allocations is:

$$
\begin{aligned}
F= & \left\{\left(y_{1}, L_{1}, y_{2}, L_{2}\right) \mid y_{1} \geq 0, y_{2} \geq 0,1 \geq L_{1} \geq 0,1 \geq L_{2} \geq 0,\right. \\
& \left.p y_{1}+(1-p) y_{2} \leq p L_{1}+(1-p) \theta L_{2}\right\} .
\end{aligned}
$$

We consider only symmetric allocations and policies-ones which treat all members of a type identically. An allocation in $F$ is Pareto efficient if there is no other allocation in $F$ that renders at least one of the types better off without diminishing the utility of either type. Denote the set of Pareto efficient allocations by $P$.

A tax policy is a vector $\tau=\left(y_{1}, x_{1}, y_{2}, x_{2}\right) \in \mathrm{R}^{4}$ where the taxing authority intends that the low (high) type will choose to produce income $x_{1}\left(x_{2}\right)$ and will receive as postfisc income, $y_{1}\left(y_{2}\right)$. Tax policies must be incentive compatible to be attainable. An attainable tax policy, $\tau$, satisfies:

(F1) $\left(y_{1}, x_{1}, y_{2}, \frac{x_{2}}{\theta}\right) \in F$ (feasibility)

(IC2) $u\left(y_{2}, \frac{x_{2}}{\theta}\right) \geq u\left(y_{1}, \frac{x_{1}}{\theta}\right)$ (incentive compatibility, high agent)

(IC1) if $x_{2} \leq 1$, then $u\left(y_{1}, x_{1}\right) \geq u\left(y_{2}, x_{2}\right)$ (incentive compatibility, low agent)

(P1) $u\left(y_{1}, x_{1}\right) \geq 0$ (participation constraint, low agent)

The (unstated) participation constraint for the high agent follows from (IC2) and (P1). We denote the set of attainable policies by $A$. Trivially, we can write $A$ as the

\footnotetext{
1 In my formulation, it would involve a Nash equilibrium between two players, in which part of the strategy of each player requires solving a Mirrleesian optimal tax problem.

2 This corresponds to the common assumption that the median wage is less than the mean wage.
} 
union of two sets, $A=A_{1} \cup A_{2}$ where:

$$
A_{1}=\left\{\tau \mid x_{2}>1\right\}, \quad A_{2}=\left\{\tau \mid x_{2} \leq 1\right\} .
$$

In general, neither of the sets $A_{1}$ or $A_{2}$, nor their union, is convex.

We say a tax policy (attainable or not) is Pareto efficient if the allocation it would induce, were it implemented, is Pareto efficient. We will also, hopefully without confusion, denote the set of Pareto efficient tax policies by $P$. We say a tax policy is second-best efficient (SBE) if it is in $A$ and it is not Pareto-dominated by another policy in $A$. Denote the set of SBE policies by $P(A)$.

Denote by $L(\omega)$ the optimal labor supply of type $\omega(\omega=1, \theta)$ in the laissez-faire economy, ${ }^{3}$ that is:

$$
L(\omega)=\underset{L}{\arg \max }(\omega L-g(L)) .
$$

We will study below what we call the interior case, in which:

(I1) $0<L(\omega)<1$, for $\omega \in\{1, \theta\}$

(I2) $\theta L(\theta)>1$.

(I2) tells us that if a tax policy specifies that $x_{2}=\theta L(\theta)$, then the unskilled agent cannot possibly imitate the skilled one, and so the constraint (IC1) is nugatory.

Of course it follows from (I1) that we have

$$
g^{\prime}(L(\omega))=\omega, \quad \omega \in\{1, \theta\}
$$

\section{Voting and political equilibrium}

A tax policy is a menu, where each worker may choose either of the proposed preand post-fisc income pairs. The utility of voters over policies in $A$ is defined in the obvious way. Denote the indirect utility function of a voter of type $\omega$ on the policy space $A$ by $v(\cdot ; \omega)$. Then

$$
v\left(y_{1}, x_{1}, y_{2}, x_{2} ; \theta\right)=y_{2}-g\left(\frac{x_{2}}{\theta}\right) \quad \text { and } \quad v\left(y_{1}, x_{1}, y_{2}, x_{2} ; 1\right)=y_{1}-g\left(x_{1}\right) .
$$

Of course, we use here the fact that the worker chooses the part of the policy menu which gives him or her greater utility.

There are two political parties, which we denote $H$ and $L$, and which represent high and low type citizens, respectively. We assume that, when facing two policies $\tau^{L}, \tau^{H}$ proposed by parties $L$ and $H$, a worker of type $\omega$ votes for policy $\tau^{L}$ when the following inequality holds:

$$
v\left(\tau^{L} ; \omega\right)>v\left(\tau^{H} ; \omega\right)+\varepsilon
$$

\footnotetext{
3 Under the laissez-faire policy, each worker keeps what she produces.
} 
where $\varepsilon$ is a random variable distributed according to a normal distribution, with mean zero, whose distribution function we denote by $N$ and whose density we denote by $n$. The stochastic term is taken to be i.i.d. across voters.

Thus, the probability that a given worker of type $\omega$ votes for $\tau^{L}$ is $N\left(v\left(\tau^{L} ; \omega\right)-\right.$ $\left.v\left(\tau^{H} ; \omega\right)\right)$. Because there is a continuum of each type, and the error term is iid, the fraction of the vote the $L$ policy will receive is

$\pi\left(\tau^{L}, \tau^{H}\right)=(1-p)\left\{\rho N\left(v\left(\tau^{L} ; 1\right)-v\left(\tau^{H} ; 1\right)\right)+N\left(v\left(\tau^{L} ; \theta\right)-v\left(\tau^{H} ; \theta\right)\right)\right\}$.

Note that, by the symmetry of the normal variate, the fraction of the vote that the $H$ policy receives is:

$$
\begin{gathered}
1-\pi\left(\tau^{L}, \tau^{H}\right)=(1-p)\left\{\rho N\left(v\left(\tau^{H} ; 1\right)-v\left(\tau^{L} ; 1\right)\right)+N\left(v\left(\tau^{H} ; \theta\right)\right.\right. \\
\left.\left.-v\left(\tau^{L} ; \theta\right)\right)\right\} .
\end{gathered}
$$

The two political parties are each managed by political entrepreneurs, who belong to one of two factions: the Opportunists or the Guardians. These factions bargain with each other in the face of the other party's policy proposal. We represent this bargaining as follows: the Opportunists choose a policy to maximize the vote share of their party, subject to a constraint which the Guardians insist upon, that the policy not deliver too low a utility to those citizens whom the party represents.

Definition A political equilibrium (PE) is a pair of policies $\left(\tau^{L}, \tau^{R}\right) \in A^{2}$ and a pair of numbers $\left(k^{L}, k^{H}\right) \in \mathrm{R}^{2}$ such that:

(E1a) $\tau^{L}$ solves the program

$$
\begin{aligned}
& \max _{\tau \in A} \pi\left(\tau, \tau^{H}\right) \\
& \text { subj. to } v(\tau ; 1) \geq k^{L}
\end{aligned}
$$

(E1b) the constraint in the program in (E1a) binds;

(E2a) $\tau^{H}$ solves the program

$$
\begin{aligned}
& \max _{\tau \in A}\left(1-\pi\left(\tau^{L}, \tau\right)\right) \\
& \text { subj. to } v(\tau ; \theta) \geq k^{H}
\end{aligned}
$$

(E2b) the constraint in program (E2a) binds;

(E3) $v\left(\tau^{L} ; 1\right) \geq v\left(\tau^{H} ; 1\right)$ and $v\left(\tau^{H} ; \theta\right) \geq v\left(\tau^{L} ; \theta\right)$. 
Condition (E1a) says that the policy $\tau^{L}$ is the outcome of efficient bargaining between the Opportunists and Guardians in Party $L$; (E2a) makes the analogous statement for party $H$; and (E3) says that each party's policy is favored by its constituency. The conditions (E1b) and (E2b) simply say that the bargaining between the factions is tight.

We have:

Proposition 1 If $\left(\tau^{L}, \tau^{H}\right)$ is a political equilibrium, then $\left(\tau^{L}, \tau^{H}\right) \in P(A) \times P(A)$.

Proof Suppose that one policy in a PE is not SBE, say $\tau^{L}$. Moving to a Paretodominating policy will increase the objective function of the $L$ party, which is given in (3.1a), without violating the $k^{L}$ constraint.

Remark Suppose we assumed a continuum of worker types. We could formulate a definition of political equilibrium with two parties, each representing the two elements in a partition of the type space. It is natural, in this case, that the Guardians attempt to maximize some weighted average of the utility of their constituents. This, indeed, is the concept of PUNE in the above-cited literature. We could thus characterize the bargaining problem within each party as the maximization of the constituents' average utility subject to a constraint that the vote share not fall below a specified level. Thus, each party would be solving a 'constrained' Mirrlees tax problem, given the tax policy proposed by the other party. This is an intractable problem, at least for a general analysis. This remark motivates our decision to study the two-type world.

\section{The Pareto set and the second-best efficient set}

From Proposition 1, it is clear that to characterize the set of equilibria, it will be helpful to understand the SBE set of policies.

First, we characterize the set of efficient policies, attainable or not, $P$.

\section{Proposition 2}

$$
\begin{aligned}
P= & \left\{\left(y_{1}, x_{1}, y_{2}, x_{2}\right)=\left(L(1)+\frac{\delta}{\rho}, L(1), \theta L(\theta)-\delta, \theta L(\theta)\right)\right. \text {, where } \\
& -\rho L(1) \leq \delta \leq \theta L(\theta)\} .
\end{aligned}
$$

In other words, the Pareto set is very simple: each worker type produces his optimal laissez-faire income; then each high type is taxed $\delta$ and each low type receives the transfer $\delta / \rho$, where $\delta$ lies in a given interval. The simplicity of the Pareto set is the purchase of quasi-linearity.

Proof 1. Suppose $\tau$ is a feasible (not necessarily attainable) policy and $x_{2} \neq \theta L(\theta)$. Construct a new policy by changing $x_{2}$ in the direction of $\theta L(\theta)$, and giving the skilled agent the extra income so generated (assuming $x_{2}<\theta L(\theta)$ ), or reducing his income by the amount of income lost (if $x_{2}>\theta L(\theta)$ ). This change will increase the skilled agent's utility_by quasi-linearity — and it will not affect the utility of 
the low agent. ${ }^{4}$ Hence $\tau$ was not Pareto efficient. In like manner, we prove that $x_{1}=L(1)$.

2. The restriction on $\delta$ merely says that each agent must receive non-negative income.

3. The budget constraint is embodied in the way the transfer is formulated.

4. Conversely, it is clear that any policy in $P$ is efficient: for production is fixed, and any way of allocating total output between the agents is therefore efficient. Any other feasible policy would have to change one of the agents' labor supplies, and we have shown such an allocation cannot be efficient.

We may now characterize the SBE set of policies. First, define $\delta=\rho(g(L(1))-$ $L(1)), \quad \bar{\delta}=p R, \quad R=\theta L(\theta)-g(L(\theta))+g\left(\frac{L(1)}{\theta}\right)-L(1)$ and let $x_{1}^{*}$ be the solution of the equation $p+\frac{1-p}{\theta} g^{\prime}\left(\frac{x}{\theta}\right)=g^{\prime}(x)$.

Theorem 15 Suppose $g^{\prime}(0)<\frac{p \theta}{\theta+p-1}$. Then $P(A)=B_{1} \cup B_{2}$ where

$$
\begin{aligned}
& B_{1} \subset P \text { and } B_{2}=P(A) \backslash P, \text { where } \\
& B_{1}=\left\{\tau \in P \mid y_{1}=L(1)+\frac{\delta}{\rho}, y_{2}=\theta L(\theta)-\delta, \text { for } \delta \leq \delta \leq \bar{\delta}\right\},
\end{aligned}
$$

and

$$
\begin{aligned}
B_{2}= & \left\{\left(y_{1}, x_{1}, y_{2}, x_{2}\right) \mid x_{2}=\theta L(\theta), x_{1}^{*} \leq x_{1} \leq L(1), \quad \text { and } \quad\left(x_{1}, y_{1}, y_{2}\right)\right. \text { solves. } \\
& \text { the system }\left\{p x_{1}+(1-p) x_{2}=p y_{1}+(1-p) y_{2}, \quad y_{2}-g\left(L((\theta))=y_{1}\right.\right. \\
& \left.\left.-g\left(\frac{x_{1}}{\theta}\right)\right\}\right\} .
\end{aligned}
$$

Theorem 1 states that $P(A)$ comprises part of the Pareto set of policies plus a one-parameter set of attainable policies in which the skilled agent continues to work at his optimal laissez-faire production, but the unskilled worker works less than his laissez-faire optimal level.

It is helpful to visualize the utility possibilities of the second-best efficient set, which is depicted in Fig. 1. The origin of the space in the figure is the utility pair generated at the laissez-faire allocation. Marks on the axes of the figure indicate deviations from the laissez-faire utilities. The policies in $B_{1}$ generate the straight line segment $\overline{a b}$; policies on the segments $\overline{b \beta}$ and $\overline{a \alpha}$ are efficient but unattainable; policies on the arc $\widehat{a c}$ are those in $B_{2}$. These are SBE but not efficient.

Proof of theorem 1: 1. First, we show that $B_{1}=P \cap A$. Since $x_{2}=\theta L(\theta)>1$, the unskilled agent cannot imitate the skilled agent at an efficient policy. It follows that $P \cap A$ is the intersection of $P$ with the set of policies such that:

$$
\text { (IC2) } \theta L(\theta)-\delta-g(L(\theta)) \geq L(1)+\frac{\delta}{\rho}-g\left(\frac{L(1)}{\theta}\right) \text {, }
$$

\footnotetext{
${ }^{4}$ For instance, if $x_{2}<\theta L(\theta)$ then note that $\theta \varepsilon-g\left(\frac{x_{2}}{\theta}+\varepsilon\right)$ is increasing for small positive $\varepsilon$, because $\theta>g^{\prime}\left(\frac{x_{2}}{\theta}\right)$, by convexity of $g$ and Eq. (2.1).

5 The premise of the theorem serves to simplify the characterization of $P(A)$; if it is dropped, the qualitative results do not change, by the statement becomes more complex.
} 


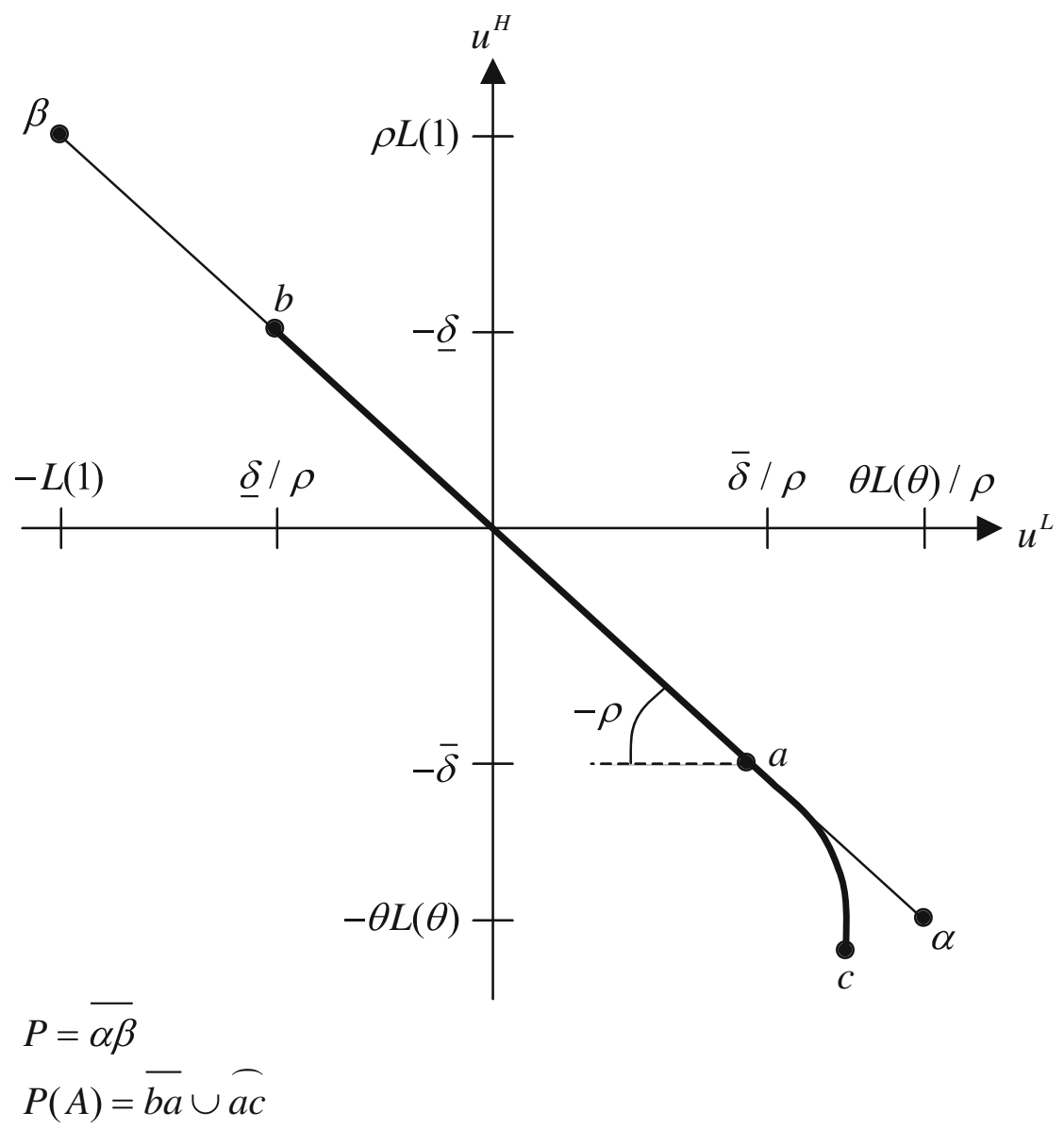

Fig. 1 Frontier of the utility possibilities set

(P1) $L(1)+\frac{\delta}{\rho}-g(L(1)) \geq 0$.

These two constraints immediately imply that $\delta \leq \delta \leq \bar{\delta}$, proving the claim of this step.

2. There are no attainable policies with the utility of the unskilled agent less than he receives at the policy defined by $\delta$, because his utility there is zero.

3. The policies in $B_{2}$ are SBE but not efficient. Consider the set of such policies, which we must establish is non-empty. We observe that at any such policy, we must have $x_{2}=\theta L(\theta)$. For suppose not: then alter $x_{2}$ to $\theta L(\theta)$, and give (or take) the changed income so generated to the skilled agent. This increases the skilled agent's utility and does not alter the utility of the unskilled agent. Moreover, the new policy is attainable. Hence the original policy was not SBE.

Therefore, if $S B E \backslash P \neq \emptyset$, then in any such policy, $x_{1} \neq L(1)$. I claim that (IC2) binds at such a policy: for suppose to the contrary that 


$$
y_{2}-g(L(\theta))>y_{1}-g\left(\frac{x_{1}}{\theta}\right) .
$$

Then alter $x_{1}$ a small amount in the direction of $L(1)$ and give (take) the resultant change in income to (from) the unskilled agent. (IC2) will still be slack, and the new policy is therefore attainable but Pareto dominates the given one.

4. It follows that any policy in $S B E \backslash P$ is of the form $\left(y_{1}, x_{1}, y_{2}, \theta L(\theta)\right)$ where

$$
\begin{aligned}
& p x_{1}+(1-p) \theta L(\theta)=p y_{1}+(1-p) y_{2}, \text { and } \\
& y_{2}-g(L(\theta))=y_{1}-g\left(\frac{x_{1}}{\theta}\right) .
\end{aligned}
$$

We may view this as a one-parameter family of policies parameterized by $x_{1}$. Viewing $y_{1}$ and $y_{2}$ as functions of $x_{1}$ we compute from (4.1) and (4.2), invoking the implicit function theorem, that:

$$
\begin{aligned}
& \frac{d y_{1}}{d x_{1}}=p+\frac{(1-p)}{\theta} g^{\prime}\left(\frac{x_{1}}{\theta}\right) \\
& \frac{d y_{2}}{d x_{1}}=p\left(1-\frac{1}{\theta} g^{\prime}\left(\frac{x_{1}}{\theta}\right)\right) .
\end{aligned}
$$

5. We write the utility of the unskilled agent at such a policy as

$$
U\left(x_{1}\right)=y_{1}-g\left(x_{1}\right)
$$

and so

$$
\frac{d U}{d x_{1}}(L(1))=p+\frac{1-p}{\theta} g^{\prime}\left(\frac{L(1)}{\theta}\right)-1=(1-p)\left(\frac{1}{\theta} g^{\prime}\left(\frac{L(1)}{\theta}\right)-1\right)
$$

which uses the fact that $g^{\prime}(L(1))=1$. Now note that by (I2), $\theta L(\theta)>L(1)$, and by the convexity of $g, \quad \theta=g^{\prime}(L(\theta))>g^{\prime}\left(\frac{L(1)}{\theta}\right)$, implying that $1>\frac{1}{\theta} g^{\prime}\left(\frac{L(1)}{\theta}\right)$. Therefore, $\frac{d U^{1}}{d x_{1}}(L(1))<0$. Consequently, reducing $x_{1}$ at $L(1)$ along the path described by (4.1) and (4.2) will indeed increase the utility of the low agent, while decreasing the utility of the high agent. Thus, the points so generated are SBE.

6. How far can $x_{1}$ be decreased? Until the utility of the unskilled agent reaches a maximum, which occurs when $\frac{d U\left(x_{1}\right)}{d x}=0$, that is, when

$$
p+\frac{(1-p)}{\theta} g^{\prime}\left(\frac{x_{1}}{\theta}\right)=g^{\prime}\left(x_{1}\right)
$$

This is the equation that defines $x_{1}^{*}$ just before the theorem's statement. We know that $x_{1}^{*}>0$ because $\frac{d U}{d x}(0)=p+\frac{1-p}{\theta} g^{\prime}(0)-g^{\prime}(0)$, which is positive by the inequality stated in the theorem's premise. So the claim follows from the intermediate value theorem. 


\section{Characterization of political equilibria}

Let $A\left(x_{1}\right)=1-\frac{1}{\theta} g^{\prime}\left(\frac{x_{1}}{\theta}\right), B\left(x_{1}\right)=g^{\prime}\left(x_{1}\right)-\frac{1}{\theta} g^{\prime}\left(\frac{x_{1}}{\theta}\right)$, and denote, for any pair of policies $\tau^{L}$ and $\tau^{H}, \Delta\left(\tau^{L}, \tau^{H} ; 1\right)=v\left(\tau^{L} ; 1\right)-v\left(\tau^{H} ; 1\right)$ and $\Delta\left(\tau^{L}, \tau^{H} ; \theta\right)=$ $v\left(\tau^{L} ; \theta\right)-v\left(\tau^{H} ; \theta\right)$. If $x \in\left[x_{1}^{*}, L(1)\right]$, denote the policy on the arc $\widehat{a c}$ for which $x_{1}=x$ by $\tau(x)=\left(y_{1}(x), x, y_{2}(x), \theta L(\theta)\right)$. (The functions $y_{1}(\cdot)$ and $y_{2}(\cdot)$ are given in Eqs. (4.1) and (4.2).)

In what follows, we sometimes refer to policies by their names in Fig. 1.

Theorem 2 A. Let $\tau^{H} \in P(A)$ be arbitrary, except $\tau^{H} \neq \tau\left(x_{1}^{*}\right)$. Then there is a unique value $\tilde{x}$, which depends upon $\tau^{H}$, with $x_{1}^{*}<\tilde{x}<L(1)$, such that $\left(\tau^{L}, \tau^{H}\right)$ is a political equilibrium when $\tau^{L}=\tau(x)$, any $x \in\left[x_{1}^{*}, \tilde{x}\right]$. If $\tau^{H}=\tau\left(x_{1}^{*}\right)$, then $\left(\tau\left(x_{1}^{*}\right), \tau\left(x_{1}^{*}\right)\right)$ is a political equilibrium. This exhausts the set of political equilibria.

$B$. If $\tau^{H} \neq \tau\left(x_{1}^{*}\right)$ and L plays $\tau(\tilde{x})$, where $\tilde{x}$ is defined in part $A$, then L wins more than half the votes.

\section{Proof Part A.}

1. Both parties play a policy which is SBE, by Proposition 1, and by condition (E3) of the definition of equilibrium, the $H$ party must play a policy that does not lie to the southeast of the $L$ policy (the direction refers to Fig. 1). The cases we must examine are the following:

(a) Both parties play a policy on the line segment $\overline{a b}$, and $L$ does not play $a$.

(b) $H$ plays a policy on $\overline{a b}$ and $L$ plays $a$.

(c) $H$ plays a policy on $\overline{a b}$ and $L$ plays a policy in $\widehat{a c}$.

(d) Both parties play a policy in $\widehat{a c}$.

We shall examine some of these statements; the rest yield to similar arguments.

2. Note first that if $\tau^{L}$ and $\tau^{H}$ are distinct equilbrium policies played by the two parties, then we must have $\left|\Delta\left(\tau^{L}, \tau^{R} ; \theta\right)\right| \geq \rho\left|\Delta\left(\tau^{L}, \tau^{R} ; 1\right)\right|>\left|\Delta\left(\tau^{L}, \tau^{R} ; 1\right)\right|$; this follows from Fig. 1. It therefore follows that $n\left(\Delta\left(\tau^{L}, \tau^{R} ; \theta\right)\right)<n\left(\Delta\left(\tau^{L}, \tau^{R} ; 1\right)\right)$, where $n(\cdot)$ is the density function of $N$.

3. In case (a) of Step $1,\left(\tau^{L}, \tau^{H}\right)$ is not a political equilibrium.

It will suffice to show that $L$ can increase its objective function of (E1) [see Eq. (3.1a)] by increasing the transfer to type $L$. In this case, the policies of $L$ and $H$ are each characterized by numbers $\delta^{L}$ and $\delta^{R}, \quad \delta^{L} \geq \delta^{R}$. The objective function of $L$, as a function of its policy $\delta$, may be written

$$
\rho N\left(\frac{\delta-\delta^{R}}{\rho}\right)+N\left(\delta^{R}-\delta\right) \equiv \Gamma(\delta),
$$

whose derivative at $\delta^{L}$ is $\Gamma^{\prime}\left(\delta^{L}\right)=n\left(\frac{\delta^{L}-\delta^{R}}{\rho}\right)-n\left(\delta^{R}-\delta^{L}\right)$. If $\delta^{L}>\delta^{R}$, we observe that this expression is positive, because $\rho>1$ and $n\left(\delta^{L}-\delta^{R}\right)=n\left(\delta^{R}-\delta^{L}\right)$. If $\delta^{L}=\delta^{R}$, then $\Gamma^{\prime}\left(\delta^{L}\right)=0$ and compute that $\Gamma^{\prime \prime}\left(\delta^{L}\right)=\frac{n^{\prime}(0)}{\rho}+n^{\prime}(0)=0$, and $\Gamma^{\prime \prime \prime}\left(\delta^{L}\right)=n^{\prime \prime}(0)\left(\frac{1}{\rho^{2}}-1\right)>0$, because $n^{\prime \prime}(0)<0$. Note we here employ the fact that $\rho>1$. Therefore, in either case, the claim is proved. 
4. In case (b) of Step $1,\left(\tau^{L}, \tau^{H}\right)$ is not a political equilibrium.

We study the case where $\tau^{L} \neq \tau^{R}$. In this case, $L$ proposes the transfer $\bar{\delta}$ and $H$ proposes a transfer $\delta^{R}<\bar{\delta}$. We again will show that $L$ can increase the value of its objective function by deviating onto the arc $\widehat{a c}$ which means decreasing $x_{1}$. We write $L$ 's objective function as a function of $x_{1}$ along the SBE path:

$$
\Theta\left(x_{1}\right)=\rho N\left(y_{1}\left(x_{1}\right)-g\left(x_{1}\right)-v\left(\tau^{H} ; 1\right)\right)+N\left(y_{2}\left(x_{1}\right)-g(L(\theta))-v\left(\tau^{H} ; \theta\right)\right),
$$

where the functions $y_{1}$ and $y_{2}$ are those defined in equations (4.1) and (4.2). We calculate the derivative at the point $a$, where $x_{1}=L(1)$ :

$$
\begin{aligned}
\Theta^{\prime}(L(1))= & \rho n\left(\frac{\bar{\delta}-\delta^{R}}{\rho}\right)\left\{p+(1-p) \frac{1}{\theta} g^{\prime}\left(\frac{x_{1}}{\theta}\right)-1\right\}+n\left(\delta^{R}-\bar{\delta}\right) p\left\{1-\frac{1}{\theta}\right. \\
& \left.\times g^{\prime}\left(\frac{x_{1}}{\theta}\right)\right\},
\end{aligned}
$$

where we have used the fact that $g^{\prime}(L(1))=1$, which may be simplified to read

$$
\Theta^{\prime}(L(1))=p\left\{1-\frac{1}{\theta} g^{\prime}\left(\frac{L(1)}{\theta}\right)\right\}\left\{n\left(\delta^{R}-\bar{\delta}\right)-n\left(\frac{\bar{\delta}-\delta^{R}}{\rho}\right)\right\}<0 .
$$

We have proved (see step 5 of the proof of Theorem 1) that the term in the first set of brackets on the r.h.s. is positive. The term in the second set of brackets is negative, so the derivative is negative. Therefore, if $L$ decreases the value of $x_{1}$, its objective increases - and utility increases, so the move is feasible. The claim is proved.

5. We now consider cases (c) and (d); that is, assume that $L$ plays a policy in the arc $\widehat{a c}$. Let $L$ 's policy be $\tau^{L}=\left(y_{1}, x_{1}, y_{2}, \theta L(\theta)\right)$ where $\left(y_{1}, y_{2}\right)$ are given by Eqs. (4.1) and (4.2). Fix $H^{\prime}$ 's policy at $\tau^{H}$; in this step we consider the case where $\tau^{H} \in \overline{a b}$.

The fraction of the vote going to party $L$ is

$$
\begin{aligned}
\varphi\left(x_{1}\right)= & p N\left(y_{1}\left(x_{1}\right)-g\left(x_{1}\right)-v\left(\tau^{H} ; 1\right)\right)+(1-p) N\left(y_{2}\left(x_{1}\right)-g(L(\theta))\right. \\
& \left.-v\left(\tau^{H} ; \theta\right)\right) .
\end{aligned}
$$

We differentiate $\phi$, using Eqs. (4.5) and (4.4):

$$
\begin{aligned}
\phi^{\prime}\left(x_{1}\right)= & p n\left(\Delta\left(\tau^{L}, \tau^{H} ; 1\right)\right)\left(p+\frac{1-p}{\theta} g^{\prime}\left(\frac{x_{1}}{\theta}\right)-g^{\prime}\left(x_{1}\right)\right) \\
& +(1-p) n\left(\Delta\left(\tau^{L}, \tau^{H} ; \theta\right)\right)\left(p\left(1-\frac{1}{\theta} g^{\prime}\left(\frac{x_{1}}{\theta}\right)\right)\right) \\
= & p n\left(\Delta\left(\tau^{L}, \tau^{H} ; 1\right)\right)\left(\frac{1}{\theta} g^{\prime}\left(\frac{x_{1}}{\theta}\right)-g^{\prime}\left(x_{1}\right)+p\left(1-\frac{1}{\theta} g^{\prime}\left(\frac{x_{1}}{\theta}\right)\right)\right) \\
& +(1-p) n\left(\Delta\left(\tau^{L}, \tau^{H} ; \theta\right)\right)\left(p\left(1-\frac{1}{\theta} g^{\prime}\left(\frac{x_{1}}{\theta}\right)\right)\right)
\end{aligned}
$$




$$
\begin{aligned}
= & p A\left(x_{1}\right)\left(p n\left(\Delta\left(\tau^{L}, \tau^{H} ; 1\right)\right)+(1-p) n\left(\Delta\left(\tau^{L}, \tau^{H} ; \theta\right)\right)\right) \\
& -p B\left(x_{1}\right) n\left(\Delta\left(\tau^{L}, \tau^{H} ; 1\right)\right) .
\end{aligned}
$$

Note that $A(L(1))=B(L(1))$, and so

$\phi^{\prime}(L(1))=p A(L(1))(1-p)\left(n\left(\Delta\left(\tau^{L}, \tau^{H} ; \theta\right)\right)-n\left(\Delta\left(\tau^{L}, \tau^{H} ; 1\right)\right)\right)<0$,

where the last inequality follows from the facts that $A(L(1))>0$ and $n\left(\Delta\left(\tau^{L}, \tau^{H} ; \theta\right)\right)-n\left(\Delta\left(\tau^{L}, \tau^{H} ; 1\right)\right)<0$, this last being from step 2 above. Therefore, it is surely the case that party $L$ should reduce $x_{1}$ from $L(1)$ and move onto the arc $\widehat{a c}$. This move will appeal to both its Guardians and Opportunists.

Let us now evaluate $\varphi^{\prime}\left(x_{1}^{*}\right)$. Observe that $B\left(x_{1}^{*}\right)=p A\left(x_{1}^{*}\right)$; therefore, $\varphi^{\prime}\left(x_{1}^{*}\right)=$ $p A\left(x_{1}^{*}\right)(1-p) n\left(\Delta\left(\tau^{L}, \tau^{R} ; \theta\right)\right)>0$. It follows by the intermediate value theorem that there is a value $\tilde{x}, L(1)<\tilde{x}<x_{1}^{*}$, at which $\varphi^{\prime}(\tilde{x})=0 ; \quad L$ must move at least to the policy $\tau(\tilde{x})$ on the arc $\widehat{a c}$, for this will be in the interest of both its Opportunists and Guardians. There is an appropriate condition on the second derivative of $g$ which will guarantee that the value $\tilde{x}$ is unique.

Indeed, given $\tau^{H}$, the policy $\tau^{L}(\tilde{x})=\left(y_{1}(\tilde{x}), \tilde{x}, y_{2}(\tilde{x}), \theta L(\theta)\right)$ where $\tilde{x}$ is determined by the condition that $\varphi^{\prime}(\tilde{x})=0$, that is, that:

$$
\frac{B(\tilde{x})}{A(\tilde{x})}=\frac{p n\left(\Delta\left(\tau^{L}(\tilde{x}), \tau^{H} ; 1\right)\right)+(1-p) n\left(\Delta\left(\tau^{L}(\tilde{x}), \tau^{H} ; \theta\right)\right)}{n\left(\Delta\left(\tau^{L}(\tilde{x}), \tau^{H} ; 1\right)\right)} .
$$

We have noted that such a value of $\tilde{x}$ exists.

It now follows that, in responding to $\tau^{H}, L$ must play a policy $\tau(x)$ for some $x \in\left[x_{1}^{*}, \tilde{x}\right]$, for these are precisely the policies in which there is conflict between the Opportunists and Guardians in $L$. How far into the interval $\left[x_{1}^{*}, \tilde{x}\right]$ toward $x_{1}^{*} L$ moves is determined by how high the value of $k^{L}$ is - that is, how militant the $L$ Guardians are.

To verify that $H$ 's policy is indeed an equilibrium response to $\tau^{L}(\tilde{x})$, we need only observe that to increase its vote share, $H$ would have to deviate towards $\tau^{L}(\tilde{x})$; but such a deviation would violate the constraint $\mathrm{E}(2 \mathrm{~b})$ if $k^{H}=v\left(\tau^{H} ; \theta\right)$. So any such pair $\left(\tau^{H}, \tau(x)\right)$ is a political equilibrium.

6. Now choose $\tau^{H} \in \widehat{a c}, \tau^{H} \neq \tau\left(x_{1}^{*}\right)$; let the value of $x_{1}$ at this policy be denoted $x_{1}^{H}$. Let $\tau^{L}$ be any policy on the arc $\widehat{a c}$ that lies between $\tau^{H}$ and $c$. Observe that Eq. (5.1) continues to hold. Compute, using (5.1), that $\varphi^{\prime}\left(x_{1}^{H}\right)=$ $p n(0)\left(A\left(x_{1}^{H}\right)-B\left(x_{1}^{H}\right)\right)>0$. As in step 4, we have $\varphi^{\prime}\left(x_{1}^{*}\right)<0$ : so, as in the argument of step 4, there is a (unique) value $\tilde{x} \in\left(x_{1}^{*}, x_{1}^{R}\right)$ up to which $L$ can move, increasing both its vote fraction and the utility of its Guardians (constituents). Consequently, as in step 4 , any pair $\left(\tau^{H}, \tau^{L}(x)\right)$ for $x \in\left[x_{1}^{*}, x_{1}^{H}\right]$ is a political equilibrium.

7. Finally, suppose that $\tau^{R}=c$. Then $(c, c)$ is the unique political equilibrium.

Part B. 
8. As long as $\tau^{H} \neq \tau(\tilde{x})$ in an equilibrium where $\tau^{L}=\tau(\tilde{x}), \quad H$ 's vote fraction will increase if it moves towards $\tau^{L}$. (This is verified by recapitulating the computation in step 4 with an appropriate change of notation.) The vote fraction for $H$ is thus strictly increasing on such a path, and achieves the value of one-half when $H^{\prime}$ s policy coincides with $\tau^{L}$. Hence, except in the equilibrium when both $H$ and $L$ play the same policy, $L$ wins more than half the vote.

If, however, $L$ plays a policy more redistributive than $\tau(\tilde{x})$, its vote share could fall below one-half.

Qualitatively, Theorem 2 says that in all political equilibria, party $L$ plays a policy that is more progressive, in the sense of more redistributive, than the most progressive Pareto efficient policy.

Here is an intuition for Theorem 2. The two critical premises are that (i) the low type form a majority, and that (ii) the noise in voter choice is normally distributed. Since there are more low than high types, if party $L$ transfers consumption from high types to low types, the per capita gain of each low type is less than the per capita loss of each high type. By assumption (ii), voting behavior of agents is more responsive when the utility difference between parties' offers is small. Hence, transferring consumption towards the low types increases the vote share of party $L$ (the party gains more $L$ votes than the $H$ votes it loses) as long as the proposals remain on the flat segment of the SBE set. Thus as party $L$ moves its policy along the flat segment of the SBE set towards point $a$, it wins more votes and increases its constituents' welfare. When we reach the curved segment $\widehat{a c}$, things get more complicated, because continuing to transfer from the high types to the low types is increasingly costly in terms of the high type's utility. The proof shows there is a limit to how much can be transferred to the low types before the $L$ vote share begins to fall. At a policy which is part of a PUNE, it must be the case that the party's vote share and the constituents' utility move in opposite directions. ${ }^{6}$

We note, as is usual with PUNE, that there is a 2-manifold of equilibria. We can first choose $\tau^{H}$ to be any SBE policy; this defines the number $\tilde{x}$, which then specifies the interval in which the policy $\tau^{L}$ must lie.

\section{On progressivity}

Define a policy $\tau \in P(A)$ as progressive if it is at least as redistributive as the laissezfaire policy: either $\tau \in \widehat{a c}$ or $\tau \in \overline{b a}$ and $\delta>0$. An SBE policy that is not progressive is regressive. As a tie-breaking convention, call the laissez-faire policy regressive. In Roemer (1999), conditions upon the environment were provided which implied that, with probability one, a progressive policy would win the election (where progressivity was defined for the particular [quadratic tax] policy space of that article). As I indicated in the introduction, the apparent ubiquity of progressive taxation in advanced democracies has been an animating question for political economy. Is it the case that progressive policies will always win, in the present environment?

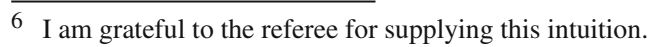


Consider the political equilibrium where party $H$ plays laissez-faire, $\tau^{H}=\tau_{L F}=$ ( $L(1), L(1), \theta L(\theta), \theta L(\theta))$ and the $L$ party plays its ideal policy, $\tau^{L}=\tau\left(x_{1}^{*}\right)$. We have:

Proposition 3 In any political equilibrium, the winning [majority] policy will be progressive if and only if the $L$ party receives at least half the vote in the contest between $\tau_{L F}$ and $\tau\left(x_{1}^{*}\right)$.

Proof The political equilibrium $\left(\tau_{L F}, \tau\left(x_{1}^{*}\right)\right)$ maximizes the vote fraction that a regressive policy can receive, over the set of political equilibria. If $H$ plays any more regressive policy, its vote share falls; if $L$ plays any less redistributive policy, its vote share rises. The claim follows.

Consequently, to decide whether progressive policies are guaranteed to win, we need only evaluate the fraction of the vote going to $L$ party in the contest between $\tau_{L F}$ and $\tau\left(x_{1}^{*}\right)$. This fraction is:

$$
\left.\Omega=p N\left(y_{1}-g\left(x_{1}^{*}\right)-L(1)+g(L(1))\right)+(1-p) N\left(y_{2}-\theta L(\theta)\right)\right)
$$

where $\left(x_{1}^{*}, y_{1}, y_{2}\right)$ is the solution of the three Eqs. (4.1), (4.2), and (4.5).

We present an example. Let $g(L)=\frac{\alpha}{2} L^{2}$, and $\theta^{2}>\alpha>\theta>1$. The postulates of the model are satisfied. We have $L(\omega)=\frac{\omega}{\alpha}, \quad \omega \in\{1, \theta\}$. Let $N$ be the distribution function of a normal variate with mean zero and standard deviation $\sigma$. We solve for $\left(x_{1}^{*}, y_{1}, y_{2}\right)$ and compute, using Mathematica, that $\Omega>0.5$ if and only if :

$$
p \operatorname{Erf}(A(p))>(1-p) \operatorname{Erf}(B(p))
$$

where $\operatorname{Erf}(\mathrm{z})=\frac{2}{\sqrt{\pi}} \int_{0}^{z} e^{-t^{2}} d t$ and $A(p)=\frac{(1-p)\left(-1+\theta^{2}\right)}{2 \sqrt{2} \alpha \sigma\left(-1+p+\theta^{2}\right)}, \quad B(p)=\frac{\rho \theta^{2}}{-1+p+\theta^{2}} A(p)$.

In Fig. 2, I graph $\Omega$ as a function of $\theta$ for various values of the parameters $(p, \alpha, \sigma)$, in each case letting $\theta$ run over the acceptable range $\theta \in(\sqrt{\alpha}, \alpha)$. We see that, in all cases, there are some values of $\theta$ for which the regressive policy wins. We furthermore see from these examples that there is no simple relationship between the mean:median wage ratio and the fraction who vote for the redistributive policy-in some cases, increasing 'skewness' reduces the support for the $L$ policy, and in others it increases it.

There thus appears to be no guarantee that, even if the median wage is less than the mean (the case in this paper), a progressive tax policy will win the election.

In the next result, we generalize from this example.

Theorem 3 Let $N$ be the normal variate with mean zero and standard deviation $\sigma$. Let $g$ be any strictly convex cost function as in our model, for which the interior case holds. Then:

A. If $p$ is sufficiently close to 0.5 (and $p>0.5$ ) then there always exist political equilibria in which a regressive policy wins.

B. If $p$ is sufficiently close to 1 , then in all political equilibria, a progressive policy wins. 

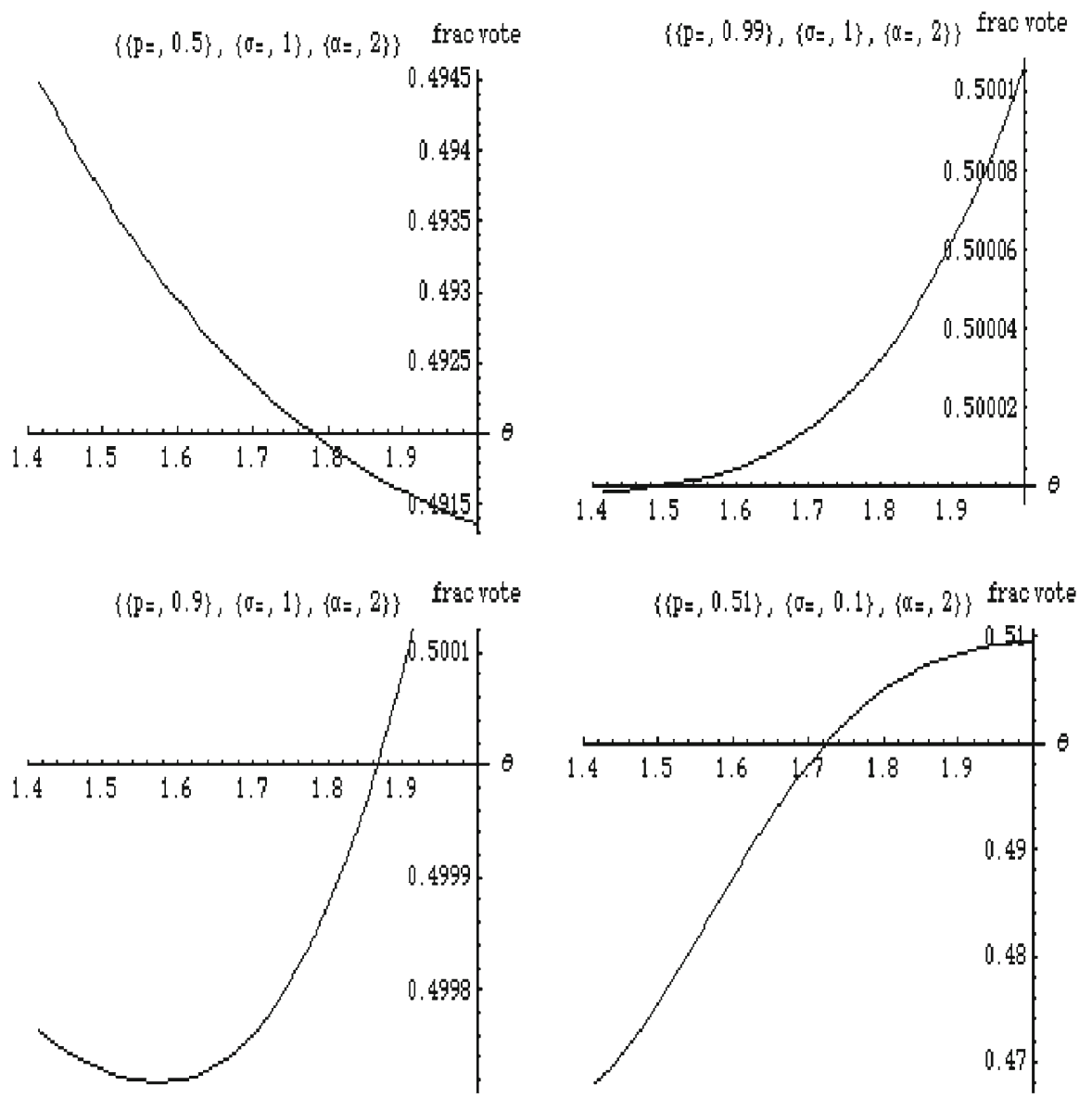

Fig. 2 The $L$ party vote fraction as a function of $\theta$ at the equilibrium $\left(\tau_{L F}, \tau\left(x_{1}^{*}\right)\right)$ when $g(L)=\frac{\alpha}{2} L^{2}$

Proof 1. For part A, we must show that, at $p=0.5$, we have $\Omega<0.5$. This condition is equivalent to:

$$
N(C)+N(D)<1
$$

where

$$
\begin{aligned}
& C=-L(1)+g(L(1))-g\left(x_{1}^{*}\right)+.5\left(x_{1}^{*}+\theta L(\theta)-g(L(\theta))+g\left(\frac{x_{1}^{*}}{\theta}\right)\right), \\
& D=.5\left(x_{1}^{*}-\theta L(\theta)+g(L(\theta))-g\left(\frac{x_{1}^{*}}{\theta}\right)\right) .
\end{aligned}
$$

(To see this, solve (4.1) and (4.2) for $y_{1}$ and $y_{2}$ in terms of $x_{1}$ and substitute into expression (6.1).) One can check that $C>0$ and $D<0$. Therefore, (6.3) is true 
iff $C+D<0$. But this last inequality is equivalent to:

$$
\frac{g(L(1))-g\left(x_{1}^{*}\right)}{L(1)-x_{1}^{*}}<1 \text {. }
$$

Recall that $g^{\prime}(L(1))=1$; since $g$ is a strictly convex function, by the theorem's premise, and $x_{1}^{*}<L(1)$, we know that $g^{\prime}$ is less than one in the interval $\left[x_{1}^{*}, L(1)\right)$. But this implies (6.4), and Part A is proved.

2. To prove Part B, we must show that $\Omega>\frac{1}{2}$ for $p$ close to 1 . Compute that at $p=1, \Omega=\frac{1}{2}$. It therefore suffices to show that $\Omega$ is decreasing as $p$ approaches one. Compute from (4.5) that, at $p=1, x_{1}^{*}=L(1)$. Evaluating the derivative:

$$
\begin{aligned}
\left.\frac{d \Omega}{d p}\right|_{p=1} & =N(0)+n(0)\left(\left.\frac{d y_{1}}{d p}\right|_{p=1}-L(1) g^{\prime}(L(1))\right)-N\left(y_{2}(1)-\theta L(\theta)\right) \\
& =\frac{1}{2}+n(0)\left(Q^{*}-L(1)\right)-N\left(Q^{*}\right)
\end{aligned}
$$

where $Q^{*}=L(1)+g(L(\theta))-g\left(\frac{L(1)}{\theta}\right)-\theta L(\theta)$. It therefore suffices to show that

$$
-n(0) Q^{*}+N\left(Q^{*}\right)>\frac{1}{2} \text {. }
$$

Note that the 1.h.s. of (6.6) equals one-half at $Q=0$, and is a decreasing function at $Q<0$. Therefore (6.6) is true, since $Q^{*}<0$, which establishes the theorem.

Part A of proposition 4 tells us that there can be no general presumption that, when the median wage is less than the mean wage (i.e., $p>0.5$ ), progressive tax policies will win elections. Part B, however, says, perhaps not surprisingly, that if the unskilled type is sufficiently numerous, then, progressivity is guaranteed.

In our model, the median wage is one (as long as $p>0.5$ ) and the mean wage is $p+(1-p) \theta$. The mean:median ratio, often called 'skewness' in the political-economy literature, is $p+(1-p) \theta$. In some models with a unidimensional policy space of affine taxes, the degree of redistribution in political equilibrium is positively correlated with skewness. It may be worth observing that Theorem 3 is a result of the opposite kind: progressivity is guaranteed when skewness is very close to one, but is not guaranteed when skewness is large (at $p=0.5$ ).

Finally, we study the effect of inequality on progressivity. The value of $\theta$, being the ratio of wages of the two types, is clearly a measure of inequality. We have:

\section{Theorem 4 Suppose that:}

(a) $g^{\prime}(0)<p$, and

(b) $\lim g(L)=\infty$.

$$
L \rightarrow 1
$$

Then for $\theta$ sufficiently large, we are in the interior case, and a progressive policy wins in all PUNEs. 
Proof 1. By assumption (a), the premise of Theorem 1 holds for all $\theta$. By assumption (b), $\lim _{L \rightarrow 1} g^{\prime}(L)=\infty$; it follows immediately that for all $\theta, L(\theta)<1$. Since $g^{\prime}(L(\theta))=\theta$, we have $L(\theta) \rightarrow 1$ as $\theta$ becomes large. It immediately follows that the conditions of interiority hold. Thus Theorems 1 and 2 are true for $\theta$ sufficiently large.

2. Consequently, to prove the progressivity claim, it suffices to verify that for large $\theta, \Omega>0.5$ : see Eq. (6.1). It clearly suffices to demonstrate that $\left(y_{1}(\theta)-g\left(x_{1}^{*}(\theta)\right)\right) \rightarrow \infty$ with $\theta$, for then the first term on the right-hand side of (6.1) will approach $p$, while the second term is non-negative. Hence the result will be proved, because $p>0.5$.

3. $x_{1}^{*}(\theta)$ is defined by the equation $p+\frac{1-p}{\theta} g^{\prime}\left(\frac{x_{1}^{*}(\theta)}{\theta}\right)=g^{\prime}\left(x_{1}^{*}(\theta)\right)$. Since $x_{1}^{*}(\theta)$ is bounded above by one, it immediately follows that $\lim _{\theta \rightarrow \infty} x_{1}^{*}(\theta)=x^{* *}$, where $x^{* *}$ is defined by the equation $g^{\prime}\left(x^{* *}\right)=p$. In particular, the derivative, $\frac{d x_{1}^{*}(\theta)}{d \theta} \rightarrow 0$ as $\theta \rightarrow \infty$.

4. We wish to compute $\frac{d}{d \theta}\left(y_{1}(\theta)-g\left(x_{1}^{*}(\theta)\right)\right)$; by step 3, we see that the second term in this expression approaches zero. Hence we are interested in $y_{1}^{\prime}(\theta)$. Differentiating Eqs. (4.1) and (4.2) implicitly w.r.t. $\theta$, using step 3, recalling that $g^{\prime}(L(\theta))=\theta$, and solving for $y_{1}^{\prime}(\theta)$, we find that:

$$
\lim _{\theta \rightarrow \infty} y_{1}^{\prime}(\theta)=1-p
$$

It therefore follows that $\lim _{\theta \rightarrow \infty} y_{1}(\theta)=\infty$. Therefore $\left(y_{1}(\theta)-g\left(x_{1}^{*}(\theta)\right)\right) \rightarrow \infty$, and the theorem is proved.

In sum, if either the unskilled type is sufficiently numerous or the skill differential is sufficiently large, then a progressive tax policy wins in political competition.

\section{Extension to more than two types}

This appears to be very difficult; it is not easy to characterize the set $P(A)$ in the threetype case. With three types, one party will represent two types-its Guardians, let us say, will seek to maximize their average utility; the other party represents the remaining type. I conjecture that in equilibrium, it is again always the case that $L$ plays a policy that is not efficient - but it must play a SBE policy (Proposition 1 continues to hold).

In the (quasi-linear) model with a continuum of types, it can be shown that the unique Pareto efficient incentive compatible policy is the laissez-faire policy. The structure of the SBE set must be quite complex.

\section{Conclusion}

In his classic work, Mirrlees analyzed tax policy from a normative viewpoint, in an environment with asymmetric information on skills. While a transition in economic practice has occurred in recent years, from the normative to the positive standpoint 
regarding government behavior, there has been little attempt to carry out the Mirrlees program in a positive model: that is, to study political-economic equilibrium over tax policy when political actors (the parties) must propose incentive compatible tax policies (an exception is De Donder and Hindriks (2003)). The present paper is a small step in such a program - albeit with two strong simplifying assumptions, to gain tractability: that there are only two types of worker, and that preferences are quasi-linear.

I have postulated, classically, that the mean wage is greater than the median wage. This is the only distributive assumption. Often, in the literature, this assumption drives a result that progressive taxation will be victorious in political competition. We characterize the set of political equilibria in tax-policy competition. It is a two dimensional manifold (this is due to our PUNE approach), in which the party representing the majority group of unskilled workers always proposes significant redistribution-in the sense that the redistribution is more extreme than any Pareto efficient redistribution. The party representing the skilled workers can propose any policy, depending on how tough its Guardian faction is.

We proved that, if the class of unskilled workers is not too large, then there always exist political equilibria in which a regressive tax policy will (almost surely) win the election. Intuitively, this will only happen if the Guardians in the $L$ party are quite powerful (that is, in the internal party bargaining game) and the Opportunists in the $H$ party are quite powerful. Thus there can be no universal presumption that democracy entails progressive taxation. However, if either the class of unskilled workers is sufficiently large, or the skill differential is sufficiently great, then progressivity is guaranteed.

Open Access This article is distributed under the terms of the Creative Commons Attribution License which permits any use, distribution and reproduction in any medium, provided the original author(s) and source are credited.

\section{References}

Boyer P, Bierbrauer F (2010) Political competition and Mirrleesian income taxation: A first pass. Max Plank Institute for Research on Collective Good, Bonn

Carbonell-Nicolau O, Klor EF (2003) Representative democracy and marginal rate progressive income taxation. J Public Econ 87:2339-2366

Carbonell-Nicolau O, Ok E (2006) Voting over income taxation. J Econ Theory. http://www.elsevier.com/ locate/jet

Casamatta G, Cremer H, De Donder P (2010) Repeated electoral competition over non-linear income tax schedules. Soc choice welf 35:535-574

De Donder P, Hindriks J (2003) The politics of progressive income taxation with incentive effects. J Public Econ 87:2491-2505

Gomberg A, Marhuenda F, Ortuño-Ortin I (2010) Endogenous party platforms: stochastic membership. Faculty of Economics, Universidad Carlos III, Madrid

Gouveia M, Oliver D (1996) Voting over flat taxes in an endowment economy. Econ Lett 50:43-50

Marhuenda F, Ortuño-Ortin I (1998) Income taxation, uncertainty and stability. J Public Econ 67:285-300

Mirrlees J (1971) An exploration in the theory of optimal taxation. Rev Econ Stud 38:175-208

Roemer J (1999) The democratic political economy of progressive taxation. Econometrica 67:1-19

Roemer J (2001) Political competition. Harvard UP, Cambridge

Roemer J (2006) Democracy, education and equality. Cambridge University Press, Cambridge

Roemer J (2006) Review of, "Positive political theory II," by D. Austen-Smith and J. Banks. J Econ Lit $44: 421-426$ 
Roemer J (2011) A theory of income taxation when parties focus upon core and swing voters. Soc choice welf. http://www.springerlink.com/content/0176-1714/preprint/?sort=p_Online Date $\&$ sortorder $=\operatorname{desc} \& o=10$ 